\title{
La Solución: Incentivo y Limitante... En La Resolución de Problemas como Estrategia en la Enseñanza de la Biología.
}

\section{The Solution: Incentive and Restriction... in Problem Solving as Strategy for Biology Teaching.}

\author{
Por: Castañeda Martín Daniel ${ }^{1}$
}

\section{Resumen}

Este escrito expone una mirada acerca de la Estrategia de Resolución de Problemas para la Enseñanza de la Biología, construida a través de la experiencia directa como estudiante en la fase de fundamentación del Programa de Licenciatura en Biología de la Universidad Pedagógica Nacional de Colombia; con él se pretende generar una reflexión acerca de la utilización de esta estrategia educativa, así como de sus implicaciones en la formación inicial de los maestros en esta área.

El tema planteado trata de resaltar, la importancia de ubicar al estudiante involucrado en los procesos de enseñanza y aprendizaje de la Biología, como actor principal de los mismos; teniendo en cuenta que, desde esta perspectiva, el estudiante es incluido con toda su experiencia de vida escolar y cotidiana, a la vez que enfatiza en la condición de sujeto inmerso en una comunidad que interviene directamente en su desarrollo.

La reflexión aquí propuesta parte de la situación contradictoria en la cual, en muchos casos, el estudiante es llevado a considerar los resultados uniformes o únicos como un requisito para dar cumplimiento y finalización de una meta propuesta o alcanzar los estándares valorativos para lograr ser promovido, pasando por alto el valor inconmensurable que tiene el proceso que transcurre en la búsqueda de los mismos. El panorama aquí descrito propone una alternativa que recupera ese valor perdido, ofreciendo oportunidades únicas en los procesos de formación.

De la misma manera esta mirada busca atribuir a los resultados e incluso al aprendizaje de los contenidos, un valor de medio más que de fin y considerar la evaluación como un proceso que contribuye a la construcción propia y colectiva de conocimiento, convirtiéndose así en una herramienta que posibilita superar falencias y procura potenciar aquellas fortalezas particulares e individuales que matizan y enriquecen a un equipo de trabajo.

Palabras claves: Resolución de Problemas- Estrategias de Enseñanza- Biología

${ }^{1}$ Licenciado en Biología UPN- 2007. Estudiante de Maestría en Educación UPN. Bogotá. Colombia2009 II. martincastaneda72@yahoo.com 


\section{Abstract}

This paper shows a view about the Problem solving strategy for Biology teaching, which is made up through direct experience as a student in the phase of fundamentation of the undergraduate program: Biology teaching degree of Universidad Pedagógica Nacional from Colombia, which it is aimed to generate a reflection upon the use of this educative strategy as well as its implications on initial teachers training in this area with.

The proposed topic tries to underline the importance of finding a way for the student that is involved in the Biology teaching and learning processes as a principal actor of these, taking into account that, from this perspective. Even the student is included with all his school of life and daily experience, at the same time it makes emphasis on the condition of subject who is immersed in a community that takes part of his development directly.

The reflection that is proposed on this paper starts from the contradictory situation in which the student is led to consider the uniform or unique results in many cases like a requirement to fulfil and finish the proposed goal or reach the valorative standards so that he can be promoted, ignoring the immensurable value that the process that takes place has for the search of these. The view that is described here, proposes an alternative that recovers that lost value, offering unique opportunities for training processes.

Likewise, this view aims to attribute a value of means rather than aim to the results and even to content learning and considers assessment like a process that contributes to the own and collective knowledge construction, becoming in this way a tool that makes possible to overcome failures and tries to boost those particular and individual strengths that clarify and enrich a job equipment.

Keywords: Problem solving - teaching strategies - Biology

\section{UN PROBLEMA CONVENIENTEMENTE PLANEADO QUE DESPIERTA EL INTERÉS DEL ESTUDIANTE}

La Resolución de Problemas como Estrategia de enseñanza de la Biología toma en cuenta las características particulares de esta Ciencia, su manera de abordar y concebir la experimentación, (la cual se diferencia a la de otras ciencias debido a su objeto de estudio); centra su atención en la potenciación de habilidades tales como, la postura crítica, la mirada holística, la capacidad de análisis, la cooperación y el trabajo en equipo. Promueve la utilización práctica de saberes de diferentes ámbitos en pro de la realización de un proceso, en el cual se propone un problema, convenientemente planeado, que despierta el interés en el estudiante y propicia las condiciones adecuadas para la apropiación de conocimientos pertinentes en la formación de esta área específica. Sigüenza, A. y Sazz, M. (1990).

Esta estrategia cuenta con etapas flexibles, no lineales que contemplan la construcción o establecimiento del problema, su presentación, comprensión y delimitación, el despliegue de las estrategias para su resolución, la comparación y análisis de los resultados, la elaboración de 
aportes y conclusiones, así como su respectiva socialización.

A pesar de lo anteriormente descrito es importante tener en cuenta que al tratarse de un proceso dinámico gestado alrededor de un sujeto de estudio vivo, es posible que el problema propuesto se transforme y sea necesario replantearlo, al igual que las estrategias sugeridas para su resolución, por lo tanto resulta ineludible realizar una evaluación continua del proceso, con el fin de establecer características y necesidades especiales, así como puntos de emergencia, propuestas innovadoras que puedan ofrecer nuevas perspectivas gestadas durante el proceso y no contempladas de manera inicial.

Es precisamente la evaluación continua del proceso, realizada con una actitud abierta, amplia, sistémica y susceptible a los cambios, la que enriquece el proceso, a la luz de esta propuesta educativa, y que a la vez repercute de manera directa en un abordaje más acorde, profundo y apropiado de las temáticas relacionadas con la Biología y su enseñanza.

\section{“.. NUESTROS PENSAMIENTOS NO TIENEN UN ORDEN... PERO AÚN LO TIENEN CUANDO LOS NECESITAMOS..."}

Esta frase recoge la impresión que suscitó a una estudiante de tercer semestre de Licenciatura en Biología, un esquema referente a la forma en la cual se enfrentan las personas a la resolución de un problema, el cual fue elaborado con la colaboración del licenciado en formación Daniel H., al finalizar cuarto semestre. Expresiones como estas evidencian de alguna manera que cualquier situación, tanto académica como cotidiana puede generar diferentes opiniones y sensaciones que pueden ser o no expresadas, pero sí tomadas en cuenta para establecer estrategias que dinamicen los procesos de enseñanza aprendizaje de la Biología.

Todo aquello que es expresado en diversas formas nos brinda una mirada al interior de la mentalidad, los sentimientos, sueños y expectativas de los estudiantes; nos permite escudriñar las maneras dinámicas en las cuales entrelazan, construyen y reconstruyen sus ideas, algunas jóvenes, rozagantes, otras arcaicas, olvidadas, unas cuantas agazapadas, otras sagaces, activas, muchas catatónicas en espera de un estímulo lo suficientemente fuerte para traerlas nuevamente a la escena y un sin número de aquellas que aún están por nacer.

Es este quizá el gran reto de aquellos que incursionan en la Resolución de Problemas como Estrategia de Enseñanza de la Biología, "encontrar" el problema adecuado que facilite el aprendizaje de aquello que resulte pertinente dentro de los requerimientos sociales y que a la vez potencie las habilidades individuales de los directamente implicados en el mismo. Desde esta perspectiva ese "encontrar" deliberadamente colocado en comillas, debe ser alcanzado a través de un proceso de construcción colectiva, en el cual el personaje es el estudiante, quien entra con todo su bagaje, tanto escolar como cotidiano y que le servirá como insumo para interpretar, delimitar e identificar el problema y de esta manera escoger las estrategias para su posible resolución.

Para la construcción del problema a trabajar se deben tener en cuenta varios aspectos, como lo mencionan Jessup y Castellanos (2002): 
- "Las personas que lo enfrentan

- El contexto socio-cultural e histórico en el cual se evidencian

- La apropiación que hace quien o quienes lo evidencian (de manera individual)

- Aquellas posibles relaciones y nuevas situaciones que pueden generarse dentro de la dinámica del mismo grupo".

La gran mayoría de los trabajos realizados sobre la Resolución de Problemas como Estrategia de Enseñanza tratan de dar claridad sobre lo que es o se puede considerar como un problema, llegando así a clasificaciones como la que realiza Frazer (1990): Problemas Reales y Artificiales, estos últimos caracterizados por tener una solución ya conocida generalmente por la persona que los presenta, y que a la vez se subdivide en cerrados si tienen una única solución o abiertos si tienen un numero variable de soluciones. Un "problema real", sería entonces, aquel del cual no se conoce la solución, incluso puede que no exista en ese momento histórico.

Mas allá del establecimiento de un consenso teórico sobre qué es un problema, se debe tener en cuenta qué es o qué puede llegar a ser un problema para el estudiante con el cual va a construirse, analizar el tema central del mismo, así como la forma que va a ser expuesto con el fin de lograr una respuesta positiva y entusiasta hacia el trabajo a realizar.

Incluir los intereses de los estudiantes, reconocer y validar sus conocimientos, ideas y sugerencias hacen parte de esta postura, que toma como base, autor y ejecutor al estudiante incluido en un grupo, pero que conserva la individualidad que enarbola la diversidad del mismo, atentando favorablemente contra la uniformidad que algunos de manera conciente o inconsciente buscan en el trabajo grupal.

Los diferentes tipos de problemas pueden ser propuestos como estrategia de enseñanza de la Biología en las instituciones educativas, sin embargo deben ser analizados cuidadosamente con el fin de establecer los fines y metas que se busca al proponerlos.

Un "problema real" puede resultar pertinente para desviar un poco el exagerado interés por la solución del mismo y dar prioridad al proceso y a las estrategias construidas o escogidas para la posible resolución; este tipo de problemas pueden llegar a incentivar la imaginación de los estudiantes y la puesta en escena de conocimientos propios de varias áreas de estudio, incluso aquellos adquiridos en ámbitos diferentes a los escolares.

Por otro lado escoger un "problema artificial" puede enfocar el trabajo en una meta enmarcada en una disciplina especifica con el fin que el estudiante se apropie de un discurso científico construido a través de situaciones problémicas, en las cuales necesita utilizar de manera adecuada los conocimientos del área abordada, para nuestro caso la Biología, logrando su integración y relación en pro de una posible resolución del problema, lo que algunos llaman "apropiación del paradigma"

Como podemos ver los diferentes tipos de problemas pueden ser utilizados de manera 
conveniente en ciertos momentos y etapas del proceso de enseñanza de la Biología y es ahí donde entra en juego la experiencia, visión y concepción que tiene el maestro sobre la educación para la puesta en marcha de la estrategia educativa.

Aquello que para algunos puede ser un "puzzle" (Problema artificial, del cual ya se conoce la respuesta) Garret (1986) para otros puede constituirse en un "problema real", haciendo la salvedad que aunque la comunidad científica, de la índole que sea, ya haya encontrado respuesta al problema, ésta es ignorada por los involucrados en el micro-mundo del aula, el cual tiene diferencias marcadas en cuanto a los propósitos y estrategias, en comparación con los espacios en los que se desarrollan trabajos de ciencia y tecnología de punta.

Es en este último aspecto donde radica la segunda parte de esta postura; propuesta que ha sido construida, y se hace necesario mencionar, a través del análisis de escritos y trabajos realizados por pedagogos e investigadores relacionados en la bibliografía que acompaña este escrito, y experiencias vividas durante mi formación escolar y particularmente en el transcurso de los primeros seis semestres de Licenciatura en Biología realizados en la Universidad Pedagógica Nacional en Bogota, por lo tanto coincide con muchos de los planteamientos expuestos por maestros, compañeros y autores sugeridos, que han caminado a mi lado en este trasegar:

\section{“...EL AFÁN POR ALCANZAR LA META DESDIBUJA LO MARAVILLOSO DEL CAMINO..."}

El plan e interés que guían el uso de esta estrategia de enseñanza deben ser claros pero flexibles, ya que muy seguramente serán influidos y transformados en el trabajo grupal, enmarcado en un contexto, que generalmente en el momento de la aplicación dista del supuesto idealizado que solo lo permite el desprevenido papel.

Es por eso que esta postura gira en torno a ver la solución del problema planteado como un medio y no como un fin, dándole así el protagonismo al proceso, a la manera de abordar analizar, asumir, relacionar y proponer estrategias de resolución, independientemente de si se consigue o no la respuesta esperada “... a veces el afán de la meta desdibuja lo maravilloso del camino".

Centrarse en la solución puede generar la posibilidad de pensar el conocimiento como un conjunto de verdades absolutas y acabadas, en espera de ser descubiertas, mientras que la perspectiva aquí propuesta trata de desestabilizar esta linealidad, valorando las respuestas diversas e invitando a emprender nuevas rutas y construcciones sobre el tema; todo ello puede redundar en la transformación de las posibles preguntas y respuestas gestadas, su descarte o respaldo, incluso hasta el punto de llegar al replanteamiento del problema mismo. De este modo, el carácter del problema lo puede constituir la forma de abordarlo, apropiarlo y las estrategias escogidas para su posible resolución, y no el planteamiento rígido del mismo o la existencia o no de las respuestas.

Desde esta postura, la educación basada en los resultados uniformes limita las posibilidades de construcción de conocimiento y coacta la imaginación y creatividad de los implicados en el 
proceso educativo, llevándolos a la mera reconstrucción, que aunque importante y valiosa mantiene a países como el nuestro, sumidos en la brecha de dependencia y consumismo que nos imponen países que ostentan el poder.

\section{LA EVALUACIÓN: HERRAMIENTA PARA POTENCIAR HABILIDADES, DETECTAR FALENCIAS Y DESPLEGAR ACCIONES RESOLUTIVAS}

Con respecto a la evaluación a aplicar en este tipo de propuestas, cabe recalcar la importancia del fin y la esencia de la misma:

La evaluación, así como la educación debe ser un fenómeno incluyente, no excluyente, con una dinámica centrípeta, que convoque, que promueva la participación y fortalezca sus bases, pero a la vez centrífuga, que proyecte, que explore nuevos puntos, e incluso supere los límites pensados de manera inicial; debe constituirse en un proceso que permita reconocer y potenciar las habilidades de los estudiantes, así como detectar las falencias en pro de establecer estrategias que logren la superación de las mismas, a través del conocimiento de las teorías científicas biológicas, pero sin anular o desvalorar las concepciones alternativas que manejan y que en muchas ocasiones resultan pertinentes para su desarrollo académico, pedagógico y psicoafectivo.

Una manera de integrar estos dos ámbitos puede ser generar conciencia en los estudiantes al resaltar la importancia de citar la fuente, el autor y/o el paradigma desde el cual elabora su discurso, señalando los puntos que apoya o refuta, así como las conclusiones propias generadas de los mismos.

Con esto se contribuye a la articulación de aquello que se debe aprender con aquello que se quiere aprender y más aún, lo que dice la ciencia, que es una de las realidades y la realidad que hace la vida más feliz, que en últimas es el fin que buscamos los humanos, aunque por distintos caminos.

\section{LA ENSEÑANZA DE LA CIENCIA DE LO VIVO: UNA TAREA ESPECIAL...}

La enseñanza de la Biología desde esta perspectiva, implica asumir una mirada holística y sistémica, que permita observar el fenómeno de lo vivo como algo solo posible en conjunción inseparable con todos los elementos que constituyen el medio en el cual se gesta y se desarrolla.

Lleva implícito en sus bases la imperiosa necesidad de asumir su objeto de estudio como algo mutable, cambiante, dinámico y complejo; requiere de un abordaje que supera las posturas de la ciencia tradicional e invita a los apasionados por la Biología a establecer procesos que promuevan la reflexión, amplíen la mirada y den cabida a nuevas posibilidades de interpretación del mundo y de sus habitantes. En este sentido, la Resolución de Problemas como Estrategia para la Enseñanza de la Biología brinda una alternativa que convoca a la cotidianidad, a la ciencia y a los sueños en un solo nicho capaz de transformar las prácticas educativas. 


\section{El reto es entonces...}

- Observar, reflexionar y construir socialmente conocimiento sobre nuestras prácticas como maestros en la enseñanza de la Biología,

- Incentivar la reflexión acerca de las características especiales del objeto de estudio de la Biología y las implicaciones que tiene el abordaje del mismo.

- Construir espacios de reflexión y construcción acerca la pertinencia de la linealidad de los métodos propuestos por la ciencia tradicional.

- Estudiar, promover y potenciar las alternativas emergentes que emanan de los procesos educativos y que procuran el crecimiento de las ciencias de la educación, así como sus estrategias de enseñanza.

- Promover la construcción social de espacios de participación en los cuales se rescate y valore la voz de los estudiantes como eje central de su formación integral.

- Reconocer en el estudiante algo más que su potencial intelectual, dando paso a las expresiones afectivas, artísticas y sociales que hacen de él un ser único e irrepetible.

\section{Y qué hacer...}

Aplicar una estrategia educativa que le permita a los estudiantes construir los elementos necesarios para poder enfrentarse a una situación problémica de manera pertinente y que a la vez promueva el reconocimiento y valoración de sus saberes propios e individuales.

\section{Cómo hacerlo...}

Teniendo en cuenta al estudiante, más allá de su ámbito escolar.

Planteando problemas contextuales y acordes con las expectativas de los estudiantes.

Estableciendo de manera clara los objetivos que deseo alcanzar mediante la aplicación de la estrategia educativa.

Asignándole un gran valor al proceso de resolución de los problemas propuestos, más que a la respuesta rígida de los mismos.

Propiciando espacios de reflexión sobre los fines, usos, metodologías, alcances y efectos del estudio y aplicación del conocimiento biológico.

\section{Qué debemos saber...}

Conocer la ciencia que voy a enseñar

Tener las habilidades para hacerlo

Y sobre todo tener claro que las ciencias son el resultado de un proceso histórico y 
dinámico, por tanto no se constituyen en verdades absolutas rígidas e inamovibles.

\section{BIBLIOGRAFÍA}

- Frazer (1990). En. Sigüenza, A. y Sazz, M. Análisis de la resolución de problemas como estrategia de enseñanza de la biología. Revista Investigación y Experiencias Didácticas, Vol. 8. No. 3, 223-230. Departamento de Biología Celular y Farmacología. Valladolid. España.

- Garret (1986) En. Sigüenza, A. y Sazz, M. (1990) Análisis de la resolución de problemas como estrategia de enseñanza de la biología. Revista Investigación y Experiencias Didácticas, Vol. 8. No. 3, 223-230. Departamento de Biología Celular y Farmacología. Valladolid. España.

- Jessup, M. \& Castellanos, R. (2002) La Resolución de Problemas como Estrategia de Educación en Ciencias Naturales. Cáp. 7. Memorias Cátedra Agustín Nieto Caballero ICFES. Universidad Pedagógica Nacional. Bogotá. Colombia.

- Sigüenza, A. y Sazz, M. (1990) Análisis de la resolución de problemas como estrategia de enseñanza de la biología. Revista Investigación y Experiencias Didácticas, Vol. 8. No. 3, 223-230. Departamento de Biología Celular y Farmacología. Valladolid. España.

“Un día caminé, otro día observé, cualquier día leí y hoy escribí... muy seguramente mañana enmendaré, pero durante toda la vida aprenderé"

\section{MartinE}

Agradecimientos a todos los maestros, compañeros y amigos que se pronuncian a través de mis palabras, en especial al Profesor Yair Porras y a mi Colega y Hermano Daniel Hernández. 